Original Article

\title{
Effects of feedback on activation of the quadriceps during weight-bearing tasks of the Wii
}

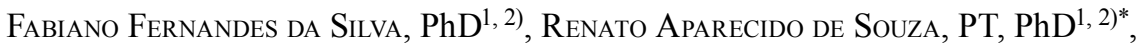 \\ Eric Fernandes Dias, PE ${ }^{2)}$, Landulfo Silveira Jr, PhD ${ }^{1)}$, Antonio Balbin Villaverde, PhD ${ }^{1)}$ \\ 1) Biomedical Engineering Institute, Camilo Castelo Branco University (UNICASTELO), Brazil \\ 2) Group of Studies and Research in Health Sciences (GEP-CS), Federal Institute of Education, Science \\ and Technology of the South of Minas Gerais (IFSULDEMINAS): 35 Caixa Postal 02, 37890-000, \\ Muzambinho, MG, Brazil
}

\begin{abstract}
Purpose] This investigation evaluated the effect of real-time feedback on electrical activation of the quadriceps during 3 weight-bearing tasks of the Wii Fit Plus ${ }^{\circledR}$. [Subjects] Thirty male healthy volunteers were recruited. [Methods] Activation of the vastus medialis, vastus lateralis, and rectus femoris muscles was recorded during virtual lunge, single leg extension, and single leg reach exercises. Each exercise was performed twice in 3 randomized experimental conditions (with visual feedback, with auditory feedback, and with no feedback). The normalized electromyographic data (using maximum voluntary isometric contraction) were analyzed using repeated measures analysis of variance and Tukey's test. [Results] No significant difference was found in the muscles among the feedback conditions during the 3 exercises. However, the variation in the muscle activity of the vastus medialis and vastus lateralis (18.23-29.20\% of maximum voluntary isometric contraction) was higher (47-62\%) than that in the rectus femoris (7.35-12.98\% of maximum voluntary isometric contraction). [Conclusion] Real-time feedback did not alter quadriceps activation during the Wii tasks. Additionally, these games showed electromyographic activation levels similar to those for the same tasks outside the virtual environment. The Wii weight-bearing tasks could therefore constitute a physical activity program but without the additional benefit of feedback.

Key words: Electromyography, Functional exercises, Exergames
\end{abstract}

(This article was submitted Jan. 5, 2015, and was accepted Feb. 14, 2015)

\section{INTRODUCTION}

Functional weight-bearing tasks (WBT) are commonly prescribed in rehabilitation for strengthening of lower extremities, especially the knee muscles ${ }^{1}$. Previous studies using electromyography (EMG) have demonstrated that lunge and squat exercises, which are classical examples of WBT, improve joint stabilization and neuromuscular control ${ }^{2)}$. However, little attention has been given to the influence of real-time feedback on the pattern of muscle activation during WBT.

Recently, a new class of video games called exergames (EXG) has used virtual reality to provide the user with perceptual and performance emulation, which has the potential for sensory ability development ${ }^{3)}$ and muscle activation ${ }^{4)}$. Various studies have used interactive virtual games from the Nintendo Wii, the world's most popular EXG, to assess their implications in the rehabilitation process and neuromuscular training ${ }^{5)}$.

\section{*Corresponding author. Renato A. Souza (E-mail:} tatosouza2004@yahoo.com.br)

(C2015 The Society of Physical Therapy Science. Published by IPEC Inc. This is an open-access article distributed under the terms of the Creative Commons Attribution Non-Commercial No Derivatives (by-ncnd) License $<$ http://creativecommons.org/licenses/by-nc-nd/3.0/>.
Considering the importance of understanding the muscle activation patterns during WBT and the influencing factors, e.g., real-time feedback, which may be helpful in several health professions, and the low cost of the Nintendo Wii technology, this study aimed to evaluate electrical activation of the quadriceps muscles during 3 WBT of the Wii Fit Plus ${ }^{\circledR}$ (lunge, single leg extension [SLE], and single leg reach [SLR]) in the following conditions: with visual feedback; with auditory feedback; and with no feedback.

\section{SUBJECTS AND METHODS}

The subject population was a convenience sample comprised of 30 male healthy volunteers recruited from the physical education faculty. Demographic data for the sample were as follows (mean \pm standard deviation [SD]): age (20 \pm 2 years $)$; body mass $(74 \pm 9 \mathrm{~kg})$; height $(175 \pm 7 \mathrm{~cm})$; fat percentage $(16 \pm 4 \%)$; lean mass percentage $(83 \pm 4 \%)$; and body mass index $\left(24 \pm 2 \mathrm{~kg} / \mathrm{m}^{2}\right)$. The following inclusion criteria were used: (a) no history of injuries of the skeletal muscle system; (b) independent in ambulation; and (c) able to perform all the experimental tasks. All participants read and signed an informed consent, and the study was approved by the Ethics Committee of the Camilo Castelo Branco University (Protocol no. 223.695). Figure 1 illustrates the design of the experimental protocol.

The virtual reality environment was simulated by the 


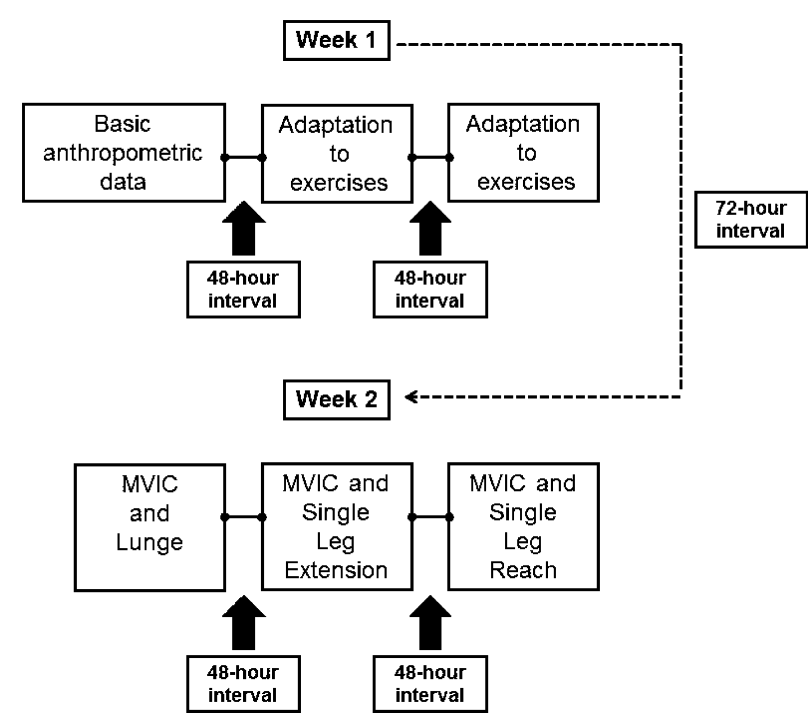

Fig. 1. Design of experimental procedures

MVIC: maximum voluntary isometric contraction

Nintendo Wii. Input devices that allowed the user-Wii interaction process were the Wii Balance Board and Sensor Bar. The Wii Balance Board has 4 pressure sensors that measure the users' body weight distribution on the lower limbs and their balance. The Wii Fit Plus ${ }^{\circledR}$ software was used, which incorporates balance, aerobic, yoga, and strengthening activities. For this study, 3 WBT of the strengthening category were chosen: lunge; SLE; and SLR (Fig. 2).

All WBT studied provide both auditory and visual real-time feedback. During the execution of these tasks, the subjects were positioned above the Wii Balance Board located $1.0 \mathrm{~m}$ from the Sensor Bar, which was attached at the base of a 42 -inch television. Each exercise was performed twice in 3 randomized experimental conditions (with visual feedback, with auditory feedback, and with no feedback), with an interval of 5 min between the tasks.

The 4-lead EMG system, Miotool 400 (Miotec, Equipamentos Biomédicos, Porto Alegre, RS, Brazil) coupled to a personal computer was used to record muscle activity. Muscle activation of the dominant limb's vastus medialis $(\mathrm{VM})$, vastus lateralis (VL), and rectus femoris (RF) was recorded for each participant using bipolar surface disc electrodes (Ag- $\mathrm{AgCl}$, Meditrace, Mansfield, MA, USA) measuring $10 \mathrm{~mm}$ in diameter, with an inter-electrode distance of $20 \mathrm{~mm}$. The EMG signals were collected using a sampling rate of 2,000 $\mathrm{Hz}$ and stored in a computer with a $14 \mathrm{bit} \mathrm{A} / \mathrm{D}$ card (Miotec, Equipamentos Biomédicos, Porto Alegre, RS, Brazil). The average EMG value of a muscle is presented as a percentage of the maximum voluntary isometric contractions (\%MVIC).

GraphPad Prism (GraphPad ${ }^{\circledR}$ Software, Inc., La Jolla, CA, USA) was used to perform the statistical analysis, with $\alpha=0.05$ set as the level of statistical significance. Comparisons were made for the \%MVIC activation of each muscle for the 3 exercises and 3 feedback conditions using repeated measures analysis of variance (ANOVA). Post hoc analyses were performed, when necessary, using Tukey's test.

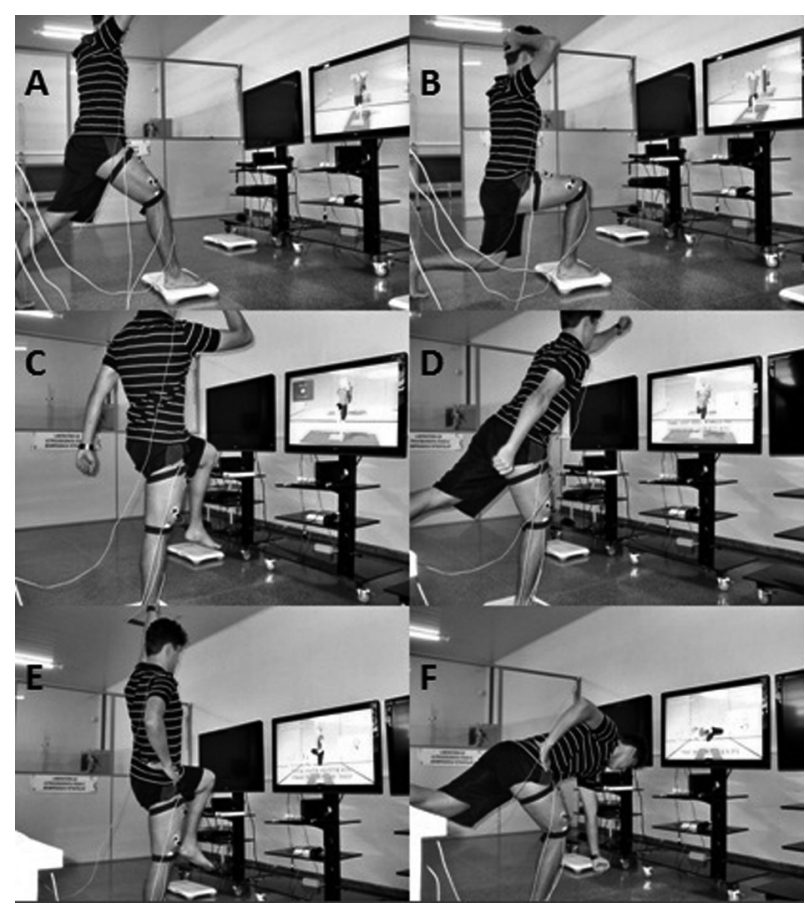

Fig. 2. Initial and final positions of the following exercises: Lunge (A and B), Single leg extension (C and D) and Single leg $\operatorname{reach}(\mathrm{E}$ and $\mathrm{F})$

\section{RESULTS}

Table 1 presents the ANOVA results (mean \pm SD of \%MVIC) for the 3 feedback experimental conditions during the lunge, SLE, and SLR exercises. There was no significant difference among the feedback conditions $(p>0.05)$ during the 3 exercises in all the muscles that were analyzed. However, there were significant differences between the EMG activation levels in RF $v s$. VL and in RF vs. VM during the 3 exercises and in all feedback conditions $(\mathrm{p}<0.05)$.

\section{DISCUSSION}

Virtual reality gaming such as the Nintendo Wii is an emerging technology that is being employed in the context of sports training and rehabilitation. Activity-promoting gaming systems are considered an attractive exercise tool perhaps due to their relative inexpensiveness, graphics that enable more enjoyable playing as well as potentially aid in adherence to physical activity programs, and generation of real-time feedback to improve task performance ${ }^{6}$. Thus, rehabilitation using real-time feedback could alter the EMG activation pattern of the quadriceps muscles, resulting in different joint movement patterns and promoting more efficient muscle activation. This hypothesis, if confirmed, could favor the incorporation of Wii games into clinical programs. Therefore, the purpose of the present study was to verify the influence of real-time feedback on EMG patterns of quadriceps muscles during WBT conducted using the Wii console, as well as the level of activation among the muscles (VM, $\mathrm{VL}$, and RF) in each exercise. To our knowledge, this is the 
Table 1. Normalized electromyographic activation during the lunge, single leg extension, and single leg reach exercises (mean $\pm \mathrm{SD})$

\begin{tabular}{llccc}
\hline Muscle & Wii-exercises & $\begin{array}{c}\text { Visual feedback } \\
\%\end{array}$ & $\begin{array}{c}\text { Auditory feedback } \\
\%\end{array}$ & $\begin{array}{c}\text { No feedback } \\
\%\end{array}$ \\
\hline Vastus medialis & Lunge & $27.1 \pm 11.4$ & $28.7 \pm 11.4$ & $29.2 \pm 12.1$ \\
& Single leg extension & $21.5 \pm 14.2$ & $24.2 \pm 15.2$ & $23.2 \pm 8.3$ \\
& Single leg reach & $20.9 \pm 12.9$ & $22.4 \pm 17.7$ & $18.6 \pm 7.4$ \\
Vastus lateralis & Lunge & $24.8 \pm 12.4$ & $26.7 \pm 14.0$ & $23.9 \pm 9.1$ \\
& Single leg extension & $21.7 \pm 14.9$ & $25.0 \pm 16.8$ & $22.4 \pm 9.0$ \\
& Single leg reach & $21.9 \pm 14.9$ & $23.1 \pm 19.7$ & $18.2 \pm 8.7$ \\
Rectus femoris & Lunge & $11.5 \pm 5.4^{\text {a }}$ & $12.2 \pm 4.8^{\text {a }}$ & $12.9 \pm 6.4^{\text {a }}$ \\
& Single leg extension & $10.0 \pm 5.0^{\text {a }}$ & $12.1 \pm 6.04^{\text {a }}$ & $12.1 \pm 6.57^{\text {a }}$ \\
& Single leg reach & $8.9 \pm 3.9^{\text {a }}$ & $9.6 \pm 5.8^{\text {a }}$ & $7.3 \pm 2.9^{\text {a }}$ \\
\hline
\end{tabular}

${ }^{a}$ indicates a difference between muscles in the same Wii-exercise and feedback condition $(\mathrm{p}<0.05)$

first study that investigated these associations. Our primary findings revealed that there are no differences among the EMG patterns generated in the visual feedback, auditory feedback, and no feedback conditions. However, considering the potential of Wii-games for entertainment, fun, and motivation, it is advantageous to include these exercises in clinical programs to strengthen the quadriceps muscle but without the additional benefit of feedback. Also, we found that the muscle activity of the VM and VL varied between $18.2-29.2 \%$ MVIC and was higher (47-62\%) than the variation in the RF, which was between 7.3-13.0\% MVIC.

Noehren, Scholz, and Davis ${ }^{7)}$ studied a group of runners with patellofemoral pain syndrome and determined that gait retraining using real-time feedback (visual feedback during 4 sessions for 1 week) was effective in reducing pain and improving hip mechanics during single-leg squats. White and Lifeso $^{8)}$ demonstrated that after 8 weeks of gait retraining using real-time feedback from an instrumented treadmill, the subjects were able to significantly improve their loading symmetry. These results suggest that motor patterns may be altered after at least 1 week of exposure to the feedback stimulus. Thus, we believed that the short-term generation of both visual and auditory feedback was insufficient to promote learning of new and more efficient motor patterns and, consequently, alter EMG activity.

In the present study, the WBT were selected based on their wide application related to practicality, functionality, and efficiency in promoting strengthening of lower limb muscles. Dynamic squatting exercises have been studied as important tools for rehabilitation programs, primarily those utilizing knee $\mathrm{EMG}^{9)}$. According to our results, the 2 vasti muscles produce approximately $50 \%$ more activity than the RF during WBT. The lower EMG activity of the RF during the WBT studied compared with that of the VM and VL muscles may be due to the biarticular function of the RF as both a hip flexor and knee extensor. Clinically, it is important to note that increased activity in the RF would increase hip flexor torque, with a concomitant increase in the activation of the antagonist muscles (e.g., hamstrings and gluteus maximus) to extend the hip, thereby altering the biomechanics of the tasks ${ }^{10}$. Another aspect that deserves mention is the similarity between the EMG activation patterns of the vasti muscles. Considering that healthy adults with normal knees show VM/VL ratios of close to $1^{9)}$, the study subjects had adequate EMG behavior for these muscles (healthy subjects). These findings could also justify the insufficiency of real-time feedback to promote additional improvements in EMG activation patterns of the quadriceps. In addition, selective strengthening of the $\mathrm{VM}$ is necessary to prevent and improve knee joint dysfunction ${ }^{11)}$. In this case, the lunge, SLE, and SLR exercises did not show any selectivity, at least in the experimental conditions studied.

There is a need for a better understanding of the EMG activation pattern strategies that occur in EXG and their implementation. In the only study found that was conducted for this purpose, Park, Lee, and Lee ${ }^{4}$ showed that EXG was an effective intervention for the muscle activities of the tibialis anterior and gastrocnemius of normal adults. For future research, we suggest investigation involving subjects with motor impairment, which could highlight a possible feedback effect, other Wii exercises with a greater degree of difficulty, and a longer duration of feedback exposure (e.g., during a training protocol).

\section{ACKNOWLEDGEMENTS}

F.F. Silva thanks Federal Institute of Education, Science and Technology of South of Minas Gerais (IFSULDEMINAS), for publication financial support and Coordination for the Improvement of Higher Education Personnel (CAPES) for doctoral scholarship. R.A. Souza thanks Fundaçao de Amparo da Pesquisa do Estado de Minas Gerais (FAPEMIG) for research grant support (grant. APQ-02660-12).

\section{REFERENCES}

1) Bouillon LE, Wilhelm J, Eisel P, et al.: Electromyographic assessment of muscle activity between genders during unilateral weight-bearing tasks using adjusted distances. Int J Sports Phys Ther, 2012, 7: 595-605. [Medline]

2) Toumi H, Best TM, Pinti A, et al.: The role of muscle strength \& activation patterns in patellofemoral pain. Clin Biomech (Bristol, Avon), 2013, 28: 544-548. [Medline] [CrossRef]

3) Barzilay O, Wolf A: Adaptive rehabilitation games. J Electromyogr Kinesiol, 2013, 23: 182-189. [Medline] [CrossRef] 
1704 J. Phys. Ther. Sci. Vol. 27, No. 6, 2015

4) Park J, Lee D, Lee S: Effect of virtual reality exercise using the nintendo wii fit on muscle activities of the trunk and lower extremities of norma adults. J Phys Ther Sci, 2014, 26: 271-273. [Medline] [CrossRef]

5) Baumeister J, Reinecke $K$, Cordes $M$, et al.: Brain activity in goal-directed movements in a real compared to a virtual environment using the Nintendo Wii. Neurosci Lett, 2010, 481: 47-50. [Medline] [CrossRef]

6) Taylor MJ, McCormick D, Shawis T, et al.: Activity-promoting gaming systems in exercise and rehabilitation. J Rehabil Res Dev, 2011, 48: 11711186. [Medline] [CrossRef]

7) Noehren B, Scholz J, Davis I: The effect of real-time gait retraining on hip kinematics, pain and function in subjects with patellofemoral pain syndrome. Br J Sports Med, 2011, 45: 691-696. [Medline] [CrossRef]
8) White SC, Lifeso RM: Altering asymmetric limb loading after hip arthroplasty using real-time dynamic feedback when walking. Arch Phys Med Rehabil, 2005, 86: 1958-1963. [Medline] [CrossRef]

9) Hyong IH, Kang JH: Activities of the vastus lateralis and vastus medialis oblique muscles during squats on different surfaces. J Phys Ther Sci, 2013, 25: 915-917. [Medline] [CrossRef]

10) Escamilla RF: Knee biomechanics of the dynamic squat exercise. Med Sci Sports Exerc, 2001, 33: 127-141. [Medline] [CrossRef]

11) Callaghan MJ, Oldham JA: Quadriceps atrophy: to what extent does it exist in patellofemoral pain syndrome? Br J Sports Med, 2004, 38: 295-299. [Medline] [CrossRef] 